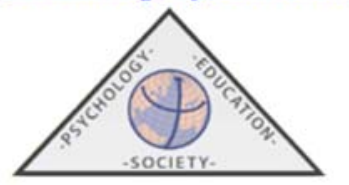

\title{
Diferencias de aprendizaje matemático entre los métodos de enseñanza ABN y CBC
}

\author{
Estíbaliz ARAGÓN, Cándida DELAGADO y Esperanza MARCHENA \\ Universidad de Cádiz, España
}

(Recibido 22 Abril, 2016; Aceptado 21 Junio, 2016)

RESUMEN: Para prevenir dificultades de aprendizaje de las matemáticas es conveniente emplear metodologías de enseñanza que se adapten al nivel y al ritmo de aprendizaje de los alumnos. Uno de estos métodos es el Algoritmo Abierto Basado en Números (ABN) que se caracteriza por ser flexible, transparente y contextualizado. El objetivo principal de este trabajo fue analizar las diferencias existentes en las habilidades matemáticas tempranas evaluadas mediante el TEMT-i entre alumnos instruidos con $\mathrm{ABN}(\mathrm{n}=68)$ y enseñanza tradicional $(\mathrm{n}=54)$. Los resultados muestran diferencias en las ganancias en los distintos componentes evaluados, siendo significativas a favor del grupo experimental en el subtest relacional, y en las habilidades numéricas de estimación y conocimiento general de los números.

Palabras clave:método ABN, sentido numérico, matemática temprana

\section{Differences in mathematical learning between $A B N$ and $C B C$ teaching methods}

\begin{abstract}
To prevent difficulties in learning mathematics is appropriate to use teaching methods that methods that fit the level and rhythm of student learning. One of these methods is the Open Algorithm Based on Numbers (ABN) which is characterized by flexibility, transparency and contextualization. The main objective of this study was to analyze the differences in early math skills assessed by TEMTt-i between students trained with ABN $(n=68)$ and traditional teaching $(n=54)$. The results show gains differences in components assessed, being significant in favor of the experimental group in relational subtest, numerical estimation skills and general knowledge of numbers.
\end{abstract}

Keywords: ABN method, number sense, early mathematics

${ }^{1}$ Correspondencia: Estibaliz Aragón, Departamento de Psicología, Universidad de Cádiz, 11510 Puerto Real-Cádiz, España Tel.: +34-956 016563; fax: +34-956 016419 Email: estivaliz.aragon@uca.es 
España Las matemáticas constituyen un pilar básico en toda sociedad, siendo fundamentales para el crecimiento económico de los países. La preocupación creciente de las naciones por esta cuestión contribuye a que se destinen recursos para su investigación, debido a la pérdida y el retroceso que supone el fracaso en dicha destreza (Hudson, Price, \& Gross, 2009; Butterworth, Varma, \& Laurillard, 2011).

Se han propuesto diversos métodos de intervención remedial para la dificultades de aprendizaje de las matemáticas, desde programas que hacen uso de las nuevas tecnologías (Aragón, Aguilar, Navarro, \& Howell, in press; Kucirkova, 2014; Räsänen, Salminen, Wilson, Aunio \& Dehaene, 2009); hasta técnicas con eficacia demostrada en este ámbito, tales como el método Copiar, Cubrir y Comparar o la Instrucción Directa con Flash Cards (Brasch, Williams, \& McLaughlin, 2008; Codding, Chan-Iannetta, Palmer, \& Lukito, 2009; Navarro \& Navarro, 2015).

En numerosas ocasiones, los investigadores han considerado e insistido en la importancia de atender a las diferencias individuales en la enseñanza, basándose en particularidades y los ritmos de aprendizaje propios de cada estudiante. Por tanto, es necesario centrar esfuerzos no sólo en proponer intervenciones específicas y remediales ante la dificultad de aprendizaje, sino también en analizar si los métodos de enseñanza que se aplican en las aulas son adecuados para el alumnado y atienden a su diversidad (Bracho \& Adamuz, 2014).

Los profesores, por lo general, emplean muchos esfuerzos, recursos y tiempo en la enseñanza de las operaciones básicas como sustrato de operaciones o cálculos más complejos o elaborados, pero sin otra finalidad ni sentido en sí mismos. En estos casos, los alumnos se limitan a memorizar instrucciones y a trabajar con cifras (Martínez-Montero, 2008). En consecuencia, no se les enseña ni se les prepara para deducir, reflexionar o extrapolar ideas y conceptos, ni se les entrena en cálculo mental ni en la estimación. Asimismo, este tipo de enseñanza suele aplicarse de manera descontextualizada, es decir, normalmente en clase de matemáticas los alumnos se limitan a resolver cuentas de manera automática sin llegar a plantearse estas operaciones en un contexto real y desproveyendo de significado a la situación de aprendizaje (Martínez-Montero, 2010).

Además de estos inconvenientes la enseñanza de las operaciones básicas mediante algoritmos tradicionales, no permite el empleo de resultados o pasos intermedios, dotando a la metodología de un carácter muy poco flexible, y dificultando el aprendizaje de los conceptos matemáticos esenciales y el desarrollo del sentido numérico, el cual se vería mucho más potenciado por otros métodos de enseñanza más abiertos y menos mecánicos (MartínezMontero, 2000, 2008).

Asimismo, además de la instrucción tradicional o cálculo basado en cifras (CBC) existen otros métodos de enseñanza de las matemáticas, que actualmente están adentrándose en nuestras aulas, como es el denominado algoritmo abierto basado en números (ABN). Este método se aplica actualmente en 13 comunidades autónomas españolas y se ha extendido a varios países de Sudamérica, tales como Argentina, México y Chile. El método ABN muestra como pilar fundamental el cálculo mental y la resolución de problemas promoviendo el aprendizaje natural de las destrezas matemáticas. Este rasgo se encuentra íntimamente relacionado con el carácter abierto del método, ya que cada estudiante puede resolver las cuestiones planteadas a su manera, en función de su nivel, permitiendo la elección de diversos cauces a la hora de abordar un mismo problema (Martínez-Montero, 2010). Este método 
permite trabajar las matemáticas de modo contextualizado, reduciendo el carácter abstracto de las matemáticas para los alumnos y contribuyendo, de este modo, a una mejora sustancial del cálculo mental, la resolución de problemas y la capacidad para estimar cantidades (Bracho, Adamuz, Gallego \& Jiménez, 2014; Bracho \& Adamuz, 2014; Martínez-Montero, 2011). Estas mejoras se deben en gran parte a la transparencia del método, que permite que el alumno emplee todos los pasos intermedios que sean necesarios a la hora de atender a las demandas del problema, de este modo, como se mencionó previamente, el mismo problema podrá resolverse de múltiples maneras con la ventaja de que la resolución de las operaciones básicas de suma y resta es válida tanto de derecha a izquierda como de izquierda a derecha.

Los estudiantes deben construir sus conceptos matemáticos sobre datos que se refieran a hechos reales, de su vida cotidiana para lograr la comprensión de las nociones matemáticas. En consecuencia, los niños y niñas mediante el método ABN son capaces de elaborar sus propios modelos formales del pensamiento a partir de otros más simples, ampliando su aprendizaje a otras áreas del conocimiento y, como consecuencia, fortaleciendo su razonamiento lógico, sin necesidad de usar únicamente su memoria como herramienta principal como ocurre con la enseñanza tradicional (Martínez-Montero, 2010).

Como se ha descrito, el uso del método ABN varía considerablemente del empleo del CBC en la enseñanza de las matemáticas. El objetivo del presente trabajo es analizar cuáles son las diferencias de rendimiento en las distintas habilidades matemáticas tempranas que se derivan del tipo de método aplicado (como variable independiente).

\section{Método}

\section{Participantes}

El presente trabajo se desarrolló a partir de una muestra de 122 estudiantes de primer curso de Educación Primaria, cuyas edades oscilaron entre los 71 y los 84 meses de edad $(M=$ 77.92; $D T=3.22)$. Del total de la muestra, 61 fueron niños $(M=77.82 ; D T=3.15)$ y 61 niñas $(M=78.02 ; D T=3.31)$. Del total de los estudiantes, 68 fueron instruidos mediante el método ABN constituyendo el grupo experimental y 54 el grupo de control cuyo aprendizaje matemático transcurrió con el método tradicional CBC.

De los 68 estudiantes instruidos mediante $\mathrm{ABN}(M=76.78 ; D T=3.30), 34$ fueron niños $(M=76.85 ; D T=3.18)$ y 34 niñas $(M=76.71 ; D T=3.46)$. Asimismo, de los 54 estudiantes que trabajaron con el método CBC $(M=79.35 ; D T=2.49), 27$ fueron niños $(M=79.04 ; D T=2.71)$ y 27 fueron niñas $(M=79.67 ; D T=2.27)$. El alumnado se seleccionó de cuatro colegios con nivel socioeconómico medio y se controló la variable tipo de colegio (público y concertado). En total en el estudio participaron alumnos que pertenecían a nueve grupos de clase, cuatro trabajaban con metodología CBC y cinco de esos grupos trabajaban con metodología ABN. Dichas aulas pertenecían a 4 colegios distintos, dos eran concertados y dos públicos. Se excluyeron del estudio aquellos alumnos que mostraban necesidades educativas especiales. 


\section{Instrumento}

Test de Evaluación Matemática Temprana-informatizado (TEMT-i) (Van Luit et al., 2015). La batería de evaluación del conocimiento matemático temprano TEMT-i informatizada y adaptada al castellano, está dirigida a la evaluación de las habilidades matemáticas tempranas. El TEMT-i está formado por dos subtests: relacional y numérico. En el primer subtest está constituido por 4 componentes: comparación, clasificación, correspondencia uno a uno y seriación. El segundo subtest consta de 5 componentes: conteo verbal, conteo estructurado, conteo (sin señalar), conocimiento general de los números y estimación. Cada uno de los componentes de cada subtest está constituido por 5 elementos, de modo que el el test completo consta de un total de 45 ítems. Cada ítem se puntúa con un punto siendo la máxima puntuación 45. Existen tres versiones del TEMT-i (A, B y C) y la batería arroja un alfa de Cronbach de .90 .

\section{Procedimiento}

Se planteó un diseño cuasi-experimental con medidas pretest y postest. El grupo experimental estuvo compuesto por aquellos alumnos que recibían instrucción mediante el método ABN desde el inicio de la etapa de Educación Infantil, el grupo control por el contrario, recibió enseñanza con metodología tradicional (CBC). Ambas metodologías se ciñen a los contenidos demandados por la administración educativa.

La investigación se llevó a cabo a lo largo de un curso escolar, al inicio del curso se realizó una medida pretest mediante el TEMT-i y al finalizar el mismo se llevó a cabo una evaluación postest. Se emplearon distintas versiones del test en cada evaluación para asegurar la fiabilidad de los resultados y que no se viesen alterados por factores relacionados con la memorización. La duración de cada sesión de evaluación rondó los 30 minutos y la administración se realizó de manera individual en aulas con condiciones óptimas para ello y durante el horario de clase.

Para realizar la investigación se obtuvo la autorización de los tutores del alumnado, así como del equipo directivo y el claustro de profesores de los colegios participantes.

Con respecto al análisis estadístico, los cálculos se realizaron con el paquete estadístico SPSS versión 22. Con dicho software se llevaron a cabo los análisis descriptivos e inferenciales. Al margen de ello, se calculó el tamaño del efecto con el fin de obtener una medida que sirviese para comparar las ganancias obtenidas en función del método de enseñanza. Con respecto al análisis inferencial, se empleó la $U$ de Mann-Whitney, una prueba no paramétrica aplicable a dos muestras independientes que se usa como alternativa a la ANOVA cuando no se cumplen los criterios para aplicarla. La finalidad de este análisis fue realizar un contraste de hipótesis que contribuyese a analizar la significación de las diferencias obtenidas. 


\section{Resultados}

Con el fin de analizar las diferencias existentes entre ambos tipos de metodología a la hora de adquirir y desarrollar las habilidades matemáticas tempranas, se calcularon los estadísticos descriptivos de cada condición experimental así como el tamaño del efecto para las distintas habilidades matemáticas tempranas. Los resultados se muestran en la tabla 1.

Tabla 1. Estadísticos descriptivos para los grupos experimental y control, al inicio y al final del curso escolar y tamaño del efecto.

\begin{tabular}{|c|c|c|c|c|c|c|c|c|}
\hline & \multicolumn{2}{|c|}{ Experimental } & \multicolumn{6}{|c|}{ Control } \\
\hline & Pretest & Postest & $d$ & $r$ & Pretest & Postest & $d$ & $r$ \\
\hline \multirow{2}{*}{ Total test TEMT-i } & 32.51 & 37.66 & 1.08 & .47 & 32.87 & 36.33 & .93 & .42 \\
\hline & $(5.76)$ & $(3.45)$ & & & $(4.06)$ & (3.29) & & \\
\hline \multirow[t]{2}{*}{ Subtest relacional } & 15.73 & 17.00 & .58 & .28 & 16.22 & 16.29 & .04 & .02 \\
\hline & $(2.48)$ & $(1.82)$ & & & $(1.76)$ & $(1.64)$ & & \\
\hline \multirow[t]{2}{*}{ Comparaciones } & 4.72 & 4.82 & .24 & .11 & 4.66 & 4.85 & .39 & .19 \\
\hline & $(.45)$ & (.38) & & & $(.58)$ & $(.35)$ & & \\
\hline \multirow[t]{2}{*}{ Clasificaciones } & 2.97 & 3.47 & .44 & .21 & 3.24 & 3.29 & .05 & .02 \\
\hline & $(1.09)$ & $(1.15)$ & & & $(1.00)$ & (.79) & & \\
\hline \multirow[t]{2}{*}{ Correspondencia } & 3.75 & 4.38 & .65 & .31 & 4.00 & 4.16 & .18 & .09 \\
\hline & $(1.11)$ & $(.79)$ & & & $(.80)$ & $(.92)$ & & \\
\hline \multirow[t]{2}{*}{ Seriación } & 4.29 & 4.32 & .03 & .01 & 4.31 & 3.98 & -.39 & -.19 \\
\hline & $(1.05)$ & (.74) & & & $(.82)$ & $(.85)$ & & \\
\hline \multirow[t]{2}{*}{ Subtest numérico } & 17.00 & 20.44 & 1.05 & .46 & 16.64 & 20.03 & 1.25 & .53 \\
\hline & (3.71) & $(2.75)$ & & & $(2.90)$ & $(2.49)$ & & \\
\hline \multirow[t]{2}{*}{ Conteoverbal } & 3.81 & 4.33 & .54 & .26 & 3.83 & 4.46 & .70 & .33 \\
\hline & $(1.09)$ & (.78) & & & $(1.05)$ & (.69) & & \\
\hline \multirow[t]{2}{*}{ Conteo estructurado } & 3.61 & 4.07 & .43 & .21 & 3.01 & 4.25 & 1.35 & .56 \\
\hline & $(1.17)$ & (.95) & & & $(1.03)$ & (.78) & & \\
\hline \multirow[t]{2}{*}{ Conteo resultante } & 3.55 & 4.25 & .67 & .31 & 3.27 & 4.27 & 1.05 & .46 \\
\hline & $(1.15)$ & $(.92)$ & & & $(1.03)$ & $(.85)$ & & \\
\hline
\end{tabular}




$\begin{array}{lcccccccc}\text { Conocimiento general } & 3.35 & 4.17 & .70 & .33 & 3.62 & 3.87 & .25 & .12 \\ \text { de los números } & (1.32) & (.99) & & & (1.03) & (.89) & & \\ \text { Estimación } & 2.66 & 3.60 & .88 & .40 & 2.88 & 3.16 & .23 & .11 \\ & (1.04) & (1.09) & & & (1.20) & (1.19) & & \end{array}$

En la tabla 1 se observan diferencias entre ambos grupos, el grupo experimental obtiene mejores puntuaciones en el postest y un mayor tamaño del efecto en el subtest relacional, así como en sus distintos componentes exceptuando las comparaciones. Por otro lado, el grupo control muestra un mayor tamaño del efecto en el subtest numérico y en las tres modalidades de conteo, sin embargo el grupo ABN supera al control en el conocimiento general de los números y estimación.

Tabla 2. Análisis descriptivo e inferencial de las ganancias obtenidas por el grupo control y experimental.

\begin{tabular}{|c|c|c|c|c|c|}
\hline & $\begin{array}{c}\text { Ganancia } \\
\text { Experimental }\end{array}$ & $\begin{array}{l}\text { Ganancia } \\
\text { Control }\end{array}$ & $d$ & $r$ & $U$ \\
\hline Total test TEMT-i & $\begin{array}{l}5.15 \\
(4.29)\end{array}$ & $\begin{array}{l}3.46 \\
(3.37)\end{array}$ & .43 & .21 & .108 \\
\hline Subtest relacional & $\begin{array}{c}1.27 \\
(2.65)\end{array}$ & $\begin{array}{c}.07 \\
(1.99)\end{array}$ & .51 & .24 & $.021^{*}$ \\
\hline Comparaciones & $\begin{array}{l}0.10 \\
(.60)\end{array}$ & $\begin{array}{c}.19 \\
(.64)\end{array}$ & -.14 & -.07 & .624 \\
\hline Clasificaciones & $\begin{array}{c}.50 \\
(1.40)\end{array}$ & $\begin{array}{c}.05 \\
(1.33)\end{array}$ & .32 & .16 & .091 \\
\hline Correspondencia & $\begin{array}{c}.63 \\
(1.24)\end{array}$ & $\begin{array}{c}.16 \\
(1.00)\end{array}$ & .41 & .20 & .066 \\
\hline Seriación & $\begin{array}{c}.03 \\
(1.29)\end{array}$ & $\begin{array}{c}-.33 \\
(1.02)\end{array}$ & .25 & .12 & .132 \\
\hline Subtest numérico & $\begin{array}{c}3.44 \\
(3.29)\end{array}$ & $\begin{array}{c}3.39 \\
(2.83)\end{array}$ & .01 & .008 & .930 \\
\hline Conteoverbal & $\begin{array}{c}.52 \\
(1.28)\end{array}$ & $\begin{array}{c}.63 \\
(1.05)\end{array}$ & -.09 & -.04 & .570 \\
\hline
\end{tabular}




$\begin{array}{lccccc}\text { Conteo estructurado } & .46 & 1.24 & -.64 & -.30 & .001^{*} \\ & (1.23) & (1.19) & & & \\ \text { Conteo resultante } & .70 & 1.00 & -.25 & -.12 & .210 \\ & (1.23) & (1.08) & & & \\ \text { Conocimiento general } & .82 & .25 & .42 & .20 & .021^{*} \\ \text { de los números } & (1.47) & (1.22) & & & \\ \text { Estimación } & .94 & .28 & .44 & .21 & .008^{*} \\ & (1.38) & (1.57) & & & \end{array}$

La tabla 2 muestra las comparaciones entre las ganancias obtenidas en las distintas habilidades matemáticas tempranas por los grupos control y experimental, pudiéndose observar como existen diferencias significativas a favor del grupo ABN en el total del subtest relacional y en las tareas de conocimiento general de los números y estimación.

Por el contrario, la ventaja es superior y significativa para el grupo control en el conteo de tipo estructurado.

\section{Discusión}

Sabemos que los tres pilares claves para el desarrollo matemático son el conocimiento del sistema de numeración convencional, el desarrollo del pensamiento lógico, y el aprendizaje significativo y contextualizado de los contenidos matemáticos (Bryant \& Nunes, 2002). La finalidad de toda metodología de enseñanza de las matemáticas es alcanzar un perfeccionamiento de la aptitud en dicha materia y por tanto del desarrollo óptimo de los tres elementos o pilares fundamentales mencionados previamente. En consecuencia, para observar la efectividad de una metodología de enseñanza-aprendizaje, es necesario centrarnos en esos tres aspectos de cara a la obtención de una competencia adecuada en matemáticas.

Son escasos los estudios que se han centrado en estudiar las diferencias existentes con respecto a las ganancias obtenidas a lo largo de un curso escolar entre el método ABN y la enseñanza CBC, siendo éste el objetivo principal del presente estudio. Los resultados derivados de esta investigación mostraron que los alumnos y alumnas instruidos con método ABN obtuvieron mejores resultados en el subtest piagetiano y en la mayoría de sus componentes, así como en las habilidades de conocimiento general de los números y estimación. Sin embargo, los estudiantes del grupo control sobresalieron en las tareas de conteo, haciéndose significativa esta diferencia en el conteo de tipo estructurado.

Greeno, Riley \& Gelman (1984) propusieron que el conteo se asienta sobre un conocimiento implícito de los principios abstractos subyacentes, posteriormente Halford (1993) sugirió que el conteo se apoyaba sobre experiencias específicas que serían utilizadas por analogía, volviendo de nuevo a la causa de la primacía de los principios primero del conteo enunciado por Gelman y Gallistel (1978). Por tanto, las actividades matemáticas requieren de 
una serie de reglas y conceptos relacionados con la noción de sentido numérico, de este modo, para un funcionamiento adecuado es necesario establecer las bases de un conocimiento interconectado y elaborado a partir de los conceptos matemáticos básicos, que va más allá de la simple memorización de información y procedimientos (Baroody, Bajwa, y Eiland, 2009).

Los resultados encontrados se deben a que los métodos de enseñanza tradicional se basan en el aprendizaje automatizado de destrezas tales como el conteo, o el cálculo mediante algoritmos tradicionales sin hacer hincapié en la comprensión.

Por tanto, los alumnos y alumnas CBC muestran menor destreza a la hora de resolver problemas que en el caso de aquellos que son capaces de dar sentido a la estrategia que están empleado para llegar a una solución adecuada (Martínez-Montero, 2010). Es por ello, que los estudiantes de enseñanza tradicional mejoran su habilidad de conteo (y en el caso de conteo estructurado difiere de forma significativa) mediante la instrucción recibida a lo largo del año, sin embargo, no extiende esa ganancia a las tareas de resolución de problemas agrupadas bajo la etiqueta conocimiento general de los números, componente del subtest numérico del TEMT-i en el que los alumnos ABN obtienen una ganancia significativamente superior.

A diferencia de los alumnos del grupo control, los estudiantes de metodología ABN dominan y desarrollan en mayor medida aquellas nociones que preceden a los conceptos numéricos como son las habilidades de tipo relacional o piagetiano, y que sirven de base a destrezas de mayor complejidad como la resolución de problemas, o que requieren mayor cantidad de recursos cognitivos y conocimiento del sistema de representaciones numéricas. Concretamente, la estimación mejora con la edad y en el caso de los niños discalcúlicos se perfecciona de manera mucho más lenta (Piazza et al., 2010). En los resultados del estudio se observa cómo los niños de enseñanza ABN muestran ventaja en el desarrollo de esta destreza, superando a los alumnos del grupo control a pesar de que en el pretest éstos mostraban puntuaciones mayores de las de los estudiantes ABN. En el postest la diferencia entre las ganancia de ambos grupos llega a ser estadísticamente significativa a favor de los alumnos ABN.

Llegados a este punto es importante llegar a plantearse si la dificultad en las matemáticas no se deriva de las diferencias individuales entre los alumnos, si no de las particularidades del método de enseñanza que se pone en práctica (Martínez-Montero, 2010). Centrándonos en esos aspectos y ofreciendo nuevo métodos que contribuyan al aprendizaje más significativo y que favorezcan una construcción óptima del pensamiento matemático, podremos prevenir posibles dificultades de aprendizaje de las matemáticas, especialmente aquellas que se derivan de método que exigen el mismo nivel de destreza a todos los alumnos.

Cabe mencionar como limitaciones del estudio el tamaño de la muestra seleccionada y la aleatoriedad de la misma. Es importante, asimismo, considerar la variable profesor a la hora de la aplicación de cada método. Por otro lado, las medidas se tomaron en primer curso de Educación Primaria sería conveniente analizar si éstas diferencias se mantienen en cursos posteriores.

\section{Agradecimientos}

Trabajo financiado por el Proyecto PSI2015-63856-P (MINECO/FEDER). 


\section{Referencias}

Aragón, E., Aguilar, M., Navarro, J.I. \& Howell, R. (in press). Improving number sense in kindergarten children with low achievement in mathematics. Annals of Psychology.

Baroody, A. J., Bajwa, N. P., \& Eiland, M. (2009). Why can't Johnny remember the basic facts?. Developmental Disabilities Research Review, 15, 69 -79. doi:10.1002/ddrr.45

Bracho, R. \& Adamuz, N. (2014). Algoritmos flexibles para las operaciones básicas como modo de favorecer la inclusión social. Revista Internacional de Educación para la Justicia Social, 3(1), 37-53.

Bracho, R., Adamuz, N., Gallego, M. C. \& Jiménez, N. (2014). Alternativa metodológica para el desarrollo integral del sentido numérico en niños y niñas de primer ciclo de educación primaria. En M. T. González, M. Codes, D. Arnau y T. Ortega (Eds.), Investigación en educación matemática XVIII (pp. 167-176). Salamanca: SEIEM.

Brasch, T. L., Williams, R. L., \& McLaughlin, T. F. (2008).The effects of a direct instruction flashcard system on multiplication fact mastery by two high school students with ADHD and ODD. Child y Family Behavior Therapy, 30(1), 51-59. doi: 10.1300/J019v30n01_04

Bryant, P. \& Nunes, T. (2002). Children's understanding of mathematics. En U. Goswami (Ed.), Blackwell handbook of childhood cognitive development (pp. 412-439). Malden: Blackwell.

Butterworth, B., Varma, S., \& Laurillard, D. (2011). Dyscalculia: from brain to education. Science, 332 (6033), 1049-1053. doi:10.1016/j.cub.2011.07.005

Codding, R. S., Chan-Iannetta, L., Palmer, M., \& Lukito, G. (2009). Examining a class wide application of Cover-Copy-Compare with and without goal setting to enhance mathematics fluency. School Psychology Quarterly, 24(3), 173. doi: http://dx.doi. org/10.1037/a0017192

Gelman, R., \& Gallistel, C. (1978). The Child's Understanding of Number. Cambridge, MA: Harvard University Press.

Greeno, J. G., Riley, M. S., \& Gelman, R. (1984). Conceptual competence and children's counting. Cognitive Psychology, 16(1), 94-143.

Halford, G. S. (1993). Children's understanding: The development of mental models. Hillsdale, NJ: Erlbaum.

Hudson, C., Price, D., \& Gross, J. (2009). The long-term costs of numeracy difficulties. London, UK: Every Child a Chance Trust.

Kucirkova, N. (2016). iRPD—A framework for guiding design-based research for iPad apps. British Journal of Educational Technology. doi:10.1111/bjet.12389

Martínez Montero, J. (2000). Una nueva didáctica del cálculo para el siglo XXI. Monografías. Escuela Española. Barcelona. Ciss Praxis.

Martínez Montero, J. (2008). Competencias básicas en matemáticas: una nueva práctica. Madrid. Wolters Kluwer.

Martínez-Montero, J. (2010). Enseñar matemáticas a estudiantes con necesidades educativas especiales. $2^{a}$ Edición. Barcelona: CISS-Praxis. 
Martínez-Montero, J. (2011). El método de cálculo abierto basado en números (ABN) como alternativa de futuro respecto a los métodos tradicionales cerrados basados en cifras (CBC). Bordón, 63(4), 95-110.

Navarro, M.J. \& Navarro, J. I. (2015). Método de intervención matemática basado en la evidencia. Revista de Psicología y Educación, 10(2),113-126.

Piazza, M., Facoetti, A., Noemi, A., Berteletti, I., Conte, S., Lucangeli, D., \& Zorzi, M. (2010). Developmental trajectory of number acuity reveals a severe impairment in developmental dyscalculia. Cognition, 116 (1), 33-41. doi: 10.1016/j.cognition.2010.03.012

Räsänen, P., Salminen, J., Wilson, A.J., Aunio, P., \& Dehaene, S. (2009). Computer-assisted intervention for children with low numeracy skills. Cognitive Development, 24, 450-472. doi:10.1016/j.cogdev.2009.09.003

Van Luit, E., Van de Rijt, B., Araújo, A., Aguilar, M., Aragón, E., Ruiz, G., ... García-Sedeño, M. (2015). Test de evaluación de la competencia matemática temprana-informatizado (TEMT-i). Madrid: EOS. 